\title{
One-Year Outcome of Aflibercept Intravitreal Injection in Vitrectomized Eyes with Diabetic Macular Edema
}

\author{
Thi Ha Chau Tran' \\ Ali Erginay ${ }^{2}$ \\ Stephane Verdun (iD) ${ }^{3}$ \\ Eric Fourmaux ${ }^{4}$ \\ Jean-François Le Rouic ${ }^{5}$ \\ Joel Uzzan (iD ${ }^{6}$ \\ Solange Milazzo ${ }^{7, \dagger}$ \\ Stephanie Baillif $\mathbb{D}^{8}$ \\ Laurent Kodjikian'

\begin{abstract}
'Service d'Ophtalmologie, Université Catholique de Lille, Faculté de Médecine et Maieutique, INSERM UI I7I, Lille, France;

${ }^{2}$ Service d'Ophtalmologie, Hôpital France; ${ }^{3}$ Département de Recherche Médicale, Groupement des Hôpitaux Catholiques de Lille, Lille, France; ${ }^{4}$ Centre Rétine Gallien, Bordeaux, France; ${ }^{5}$ Institut Ophtalmologique de l'Ouest Jules Verne, Ophtalliance, Nantes, France; ${ }^{6}$ Clinique Mathilde, Rouen, France; ${ }^{7}$ Service d'Ophtalmologie, Centre Hospitalier Universitaire d'Amiens Picardie, Université de Picardie Jules Verne, Amiens, France;

${ }^{8}$ Service d'Ophtalmologie, Centre Hospitalier Université Côte d'Azur, Université Côte d'Azur, Nice, France;

'Service d'Ophtalmologie, Croix Rousse Hospital, Hospice Cilvils de Lyon I,
\end{abstract} \\ Lariboisière APHP, Universite de Paris, Paris, \\ Université de Lyon I, Lyon, France
}

tSolange Milazzo passed away on March 23, 2019

Correspondence: Thi Ha Chau Tran Service d'Ophtalmologie, Université Catholique de Lille, Faculté de Médecine et Maieutique, INSERM UI I7I, Boulevard de Belfort, BP387, Lille, Cedex, 5902I, France

Tel +33 320877442

$\mathrm{Fax}+3320877558$

Email tran.hachau@ghicl.net
Aim: To evaluate the efficacy of intravitreal Aflibercept injection (IAI) for vitrectomized eyes with diabetic macular edema (DME) at one year.

Methods: This is a prospective, non-comparative, multicenter observational study including diabetic patients whose HbA1c is $<9 \%$, with visual acuity between 20/400 to 20/40 due to DME, who have undergone vitrectomy since at least 3 months before the first aflibercept injection. Treatment protocol included 5 monthly aflibercept injection followed by a ProReNata regimen during the first year. Visual acuity, OCT findings and number of IAI were assessed at 6 months and one year.

Results: Forty-six eyes were included. Indications for vitrectomy were epiretinal membrane (58.7\%), intravitreal hemorrhage (26.1\%), and vitreomacular traction $(8.7 \%)$, retinal detachment $(4.3 \%)$, and other cause $(4.3 \%)$. Median duration of macular edema was 3 years. Median interval between vitrectomy and first visit was 9 months. Thirty eyes were nonnaïve and received previously thermal laser (44.3\%), intravitreal injection of triamcinolone (26.7\%), of ranibizumab (70\%), of dexamethasone implant $(36.7 \%)$, or bevacizumab $(6.7 \%)$. Data was available for 35 eyes at 1 year. Visual gain was significant, +6 letters $(p<0.001)$ and central subfield thickness (CST) decreased significantly $(-108 \mu \mathrm{m}, \mathrm{p}<0.001)$ at 1 year. Mean number of injections was 9.3 and mean interval injection was 5.8 weeks.

Conclusion: These results suggest that IAI may be beneficial in vitrectomized eyes with refractory DME which require frequent injections to obtain visual and anatomical improvement.

Clinical Trial Registration: http://www.clinicaltrials.gov, registration Number NCT02874859.

Keywords: aflibercept, diabetic macular edema, DME, vitrectomy, anti-VEGF

\section{Introduction}

Diabetic macular edema (DME) is the leading cause of blindness among people of working age. Treatment of DME included blood pressure and glycemic control, grid laser, intravitreal injection of steroid (triamcinolone acetonide, dexamethasone implant, fluocinolone acetonide implant) or anti-VEGF (bevacizumab, ranibizumab, aflibercept). Anti-VEGF is considered as first line therapy. ${ }^{1}$ Several clinical trials have confirmed that Intravitreal aflibercept Injection (IAI) yields greater functional and anatomical gains than macular laser treatment of center-involving DME. ${ }^{2,3}$ The Diabetic Retinopathy Clinical Research Network (DRCR.net), protocol T, has also demonstrated that aflibercept is superior to ranibizumab or bevacizumab in eyes 
with worse vision at one year, but the significant effects were not maintained at 24 months. ${ }^{4,5}$ Aflibercept may also be useful in DME unresponsive to multiple ranibizumab/ bevacizumab injections. ${ }^{6}$

Pars plana vitrectomy (PPV) is beneficial in epiretinal membrane, ${ }^{7}$ vitreous hemorrhage, DME associated with vitreomacular traction or retinal detachment. ${ }^{8}$ Several authors have also reported good results following vitrectomy and internal limiting membrane peeling for DME without any tractional component. ${ }^{9}$ Following vitrectomy performed for DME, macula thickening was reduced in most eyes, but only one third to half of theses showed improvement of visual acuity. ${ }^{10}$ Moreover, the recurrence of macular edema requires continuous drug therapy for some patients. ${ }^{11}$

There is little information with inconsistent results in previously vitrectomized eyes with DME treated with ranibizumab or bevacizumab. ${ }^{12-15}$ In theory, the antiVEGF is likely washed away from the retina faster than when the vitreous is present, resulting to less improvement of anatomic and visual gain and the need of frequent injection. ${ }^{16}$ The purpose of this study is to investigate the efficacy of aflibercept in previously vitrectomized eyes with DME.

\section{Patients and Methods Study Design}

MODEVA (NCT02874859) was a prospective, multicenter observational cohort study of vitrectomized eyes with persistent DME treated with IAIs from November 2016 to November 2019 in 8 private and public ophthalmological clinics in France. This study was performed in accordance with the International Conference on Harmonization guidelines for Good Clinical Practice and the Helsinki Declaration. The study protocol and informed consent were reviewed and approved by the French Consultative Committee on the Processing of Information in Heath Research and by the French National Medical Council prior patient enrollment. All participants provided informed consent. Data of the study, which are available during 10 years, may be obtained up on reasonable request to the Clinical Research Department of the first's author institution. Sharable data are deidentified participants, study protocols and statistical plan.

Inclusion criteria included: 1) diabetic patients aged $\geq$ 18 years with type 1 or type 2 diabetes; 2) patients with $\mathrm{HbA} 1 \mathrm{c}<9 \%$; 3) patients with a visual acuity (VA) from
20/400 to 20/40 due to DME; and 4) patients who have undergone vitrectomy at least 3 months previously and who did not receive any treatment for DME 3 months prior first Aflibercept injection. Exclusion criteria included pregnancy and thromboembolic event since less than 3 months. Patients were recruited consecutively during a two-year period.

\section{Intervention and Observation Procedure}

Best-corrected visual acuity (BCVA) using Measurement of Early Treatment Diabetic Retinopathy Study (ETDRS) or Snellen chart converted to approximated ETDRS letter scores, intraocular pressure assessment, spectral-domain optical coherence tomography (SD-OCT), fluorescein angiography were performed at baseline. Visual acuity, SD-OCT and adverse event monitoring were recorded at each visit. Central Subfield Thickness (CST), macular volume (MV), presence of Disorganization of the Retinal Layer (DRIL), intraretinal fluid (IRF), subretinal fluid (SRF), hyper reflective foci (HRF), and disruption of ellipsoid zone (EZ) and the choroidal thickness were collected.

The IAI was administered following the recommendations of the European Medicines Agency with 5 doses of Aflibercept injection $(2 \mathrm{mg} / 0.05 \mathrm{~mL})$ at 4 weeks-intervals during the loading phase followed by a ProReNata regimen (retreatment if any fluid on OCT) during the maintenance phase. Participants were followed by a Treat and Extend or Observe and plan regimen during the second year. ${ }^{17}$ Improvement was considered to be an increase of 5 letters or a reduction at least of $10 \%$ of the CST compared to baseline at 1 year-endpoint. At each visit, BCVA by Snellen or ETDRS chart and SD-OCT have been performed. The same chart and OCT machine were used for each patient's followup for each center. Data were collected at baseline, at 6, 12 and 24 months after initiation of IAI. Window for data collection at the chosen annual time points was 2 months. Patients could withdraw from the study at any time or could be withdrawn at the discretion of the investigator for safety, behavioral or administrative reasons.

\section{Statistical Analysis}

Demographic information, medical history, and clinical characteristics were collected by electronic Case Report Form. Statistical analysis was performed with the $\mathrm{R}$ software (version 3.6.1). For the descriptive analysis, continuous variables were presented as mean \pm standard deviation, first and last quartile, minimum and maximum, 
and the categorical variable as numbers and frequencies. In the event of missing data, measurements of previous or subsequent visits were used. Change in visual acuity from baseline to 1 year was tested using Student paired $t$-test. The relationship between visual gain at 1 year and different parameters was studied using Spearman's nonparametric correlation and one way ANOVA model. Adherence to protocol and treatment changes were described. Statistical significance was set as $\mathrm{P}<0.05$.

\section{Results}

\section{Study Participants}

We included 46 previously vitrectomized eyes of 44 patients from 8 centers in France treated with aflibercept injection for DME. Demographic and clinical data are summarized in Table 1. Median age was 67.5 years [Q1Q3 59-73.5], and 59\% were male. Most patients had type 2 disease $(85 \%)$, with long duration of diabetes (median = 16 years, Q1-Q3, 11.8-25.2] and multiple comorbidities (82.6\%). Mean hemoglobin 1c (HbA1c) was 7.6\%. Lipidlowering therapy by statin treatment was given in 14 patients $(30.4 \%)$.

\section{Ocular Characteristics of Vitrectomized Eyes with DME}

Median duration of DME was 3 years (Q1-Q3, 0.5-5). PPV was performed with large variation in timing, ranging from 3 months to 20 years, with median interval of 8 months (Q1-Q3, 4-27). Internal limiting membrane peeling was performed in most cases and in most centers. All patients received first aflibercept injection 3 months after vitrectomy to avoid beneficial bias from surgery. Principal reasons of vitrectomy were DME associated with epiretinal membrane or vitreous hemorrhage. None of them had received silicone oil. We did not have detail of internal limiting membrane peeling since surgery has been done at various times and in multiple centers. When indication of surgery was vitreous hemorrhage from neovascularization, endolaser was given during all vitrectomies.

The vitrectomized eyes presented a refractory persistent DME, $30(65 \%)$ of these were non-naïve and previously received macular thermal laser (43.3\%), intravitreal steroid $(26.7 \%)$ for triamcinolone acetonide and $36.7 \%$ for dexamethasone implant), or anti-VEGF intravitreal injection (70\% for ranibizumab and $6.7 \%$ for bevacizumab). Most vitrectomized eyes were pseudophakic $(77.8 \%)$. None of them was suspected to have
Table I Baseline Characteristics of 46 Vitrectomized Eyes Receiving Aflibercept for DME

\begin{tabular}{|c|c|}
\hline Number of eyes & 46 \\
\hline Age (years), median, [QI-Q3] & $67.5[59-73.5]$ \\
\hline Gender $M / F(\%)$ & $59 / 41$ \\
\hline Type of diabetes (\% type I/\% type 2 ) & $15 / 85$ \\
\hline Duration of diabetes (years), median, [QI-Q3] & $17[11.8-26.2]$ \\
\hline HbAlc (\%), median, [QI-Q3] & $7.6[6.8-8.1]$ \\
\hline $\begin{array}{l}\text { Complications related to diabetes } \\
\text { Preexisting hypertension } \\
\text { Cardiovascular disease } \\
\text { Preexisting nephropathy } \\
\text { Preexisting peripheral neuropathy }\end{array}$ & $\begin{array}{l}33(71.7 \%) \\
8(17.4 \%) \\
8(17.4 \%) \\
4(8.7 \%)\end{array}$ \\
\hline $\begin{array}{l}\text { Ocular history } \\
\text { Duration of DME (years), median, [QI-Q3] } \\
\text { Time since vitrectomy, mean } \pm \text { SD (y), } \\
\text { median, [QI-Q3] (m) }\end{array}$ & $\begin{aligned} & 3[0.5-5] \\
2 \pm & 4.4 ; 9[3-24]\end{aligned}$ \\
\hline $\begin{array}{l}\text { Reason for vitrectomy } \\
\text { Epiretinal membrane } \\
\text { Vitreous hemorrhage } \\
\text { Vitreomacular traction } \\
\text { Retinal detachment } \\
\text { Other }\end{array}$ & $\begin{aligned} 27 & (58.7 \%) \\
12 & (26.1 \%) \\
4 & (8.7 \%) \\
2 & (4.3 \%) \\
2 & (4.3 \%)\end{aligned}$ \\
\hline Previous DME treatment & $30(65 \%)$ \\
\hline $\begin{array}{l}\text { Grid laser, } n(\%) ; \text { mean number of treatment } \\
\text { (min-max) }\end{array}$ & $\begin{array}{c}13(43.3 \%) ; 2 \\
(I-4)\end{array}$ \\
\hline Triamcinolone acetonide & $8(26.7 \%) ; 3(I-6)$ \\
\hline Ranibizumab intravitreal injection & $\begin{array}{l}2 I(70 \%) ; 7 \\
\quad(I-20)\end{array}$ \\
\hline Dexamethasone implant intravitreal injection & $\begin{array}{c}\text { II (36.7\%); } 1.5 \\
(1-4)\end{array}$ \\
\hline Bevacizumab intravitreal injection & $2(6.7 \%), 1$ \\
\hline $\begin{array}{l}\text { Ocular characteristics } \\
\text { VA (ETDRS letter score), mean (SD); median, } \\
\text { [QI-Q3] } \\
\text { Phakic /Pseudophakic }(\%) \\
\text { Intraocular pressure, }(\mathrm{mmHg}) \text {, median, } \\
\text { [Q I-Q3] }\end{array}$ & $\begin{array}{c}53.3(14.7) ; 58.6 \\
{[45-65]} \\
22.2 / 77.8 \\
16[14-17]\end{array}$ \\
\hline $\begin{array}{l}\text { DR severity on clinical examination } \\
\text { None } \\
\text { Microaneurysms only } \\
\text { Mild/Moderate NPDR } \\
\text { Severe NPDR } \\
\text { PDR } \\
\text { Previous scatter laser }\end{array}$ & $\begin{array}{c}0 \\
2(8 \%) \\
16(34.6 \%) \\
16(34.6 \%) \\
10(22.7 \%) \\
25(54.3 \%)\end{array}$ \\
\hline
\end{tabular}

(Continued) 
Table I (Continued).

\begin{tabular}{|l|c|}
\hline Macular ischemia on fluorescein angiography & \\
Mild & $13(38.2 \%)$ \\
Moderate & $14(41.2 \%)$ \\
Severe & $7(20.6 \%)$ \\
\hline
\end{tabular}

Abbreviations: M, male; F, female; DME, diabetic macular edema; ETDSR, Early Treatment Diabetic Retinopathy Study; DR, diabetic retinopathy; NPRD, nonproliferative diabetic retinopathy; PRD, proliferative diabetic retinopathy; VA, visual acuity; SD, standard deviation.

pseudophakic edema. Mean intraocular pressure was 16.5 $\pm 4.6 \mathrm{mmHg}$. The majority of eyes had proliferative diabetic retinopathy or previously had panretinal photocoagulation prior or during vitrectomy (77\%). Macular ischemia, evaluated by fluorescein angiography, according to the investigator's discretion, was considered as mild in $38.2 \%$ of eyes, moderate in $41.2 \%$ and severe in $20.6 \%$.

Baseline characteristics of SD-OCT were summarized in Table 2. Median CST was $425 \mu \mathrm{m}$ (Q1-Q3, $335-510 \mu \mathrm{m})$. Fluid was found in all eyes, distributed to intraretinal compartment in $93.3 \%$, subretinal compartment in $55.6 \%$, associated with hyperreflective foci $(84.4 \%),{ }^{18}$ Disorganization of Retinal Inner Layers (44.4\%), ellipsoid zone (EZ) disruption (44.4\%), and external limiting membrane (ELM) disruption (44.4\%). ${ }^{19}$

\section{Adherence to Treatment Protocol}

Eight pairs of eyes were dropped from the study. Withdrawal occurred in 6 cases between month 2 and

Table 2 Baseline Characteristic of SD-OCT of 46 Vitrectomized Eyes Receiving Intravitreal Aflibercept

\begin{tabular}{|l|c|}
\hline $\begin{array}{l}\text { Central subfoveal thickness }(\mu \mathrm{m}) \\
\text { Mean (SD) }\end{array}$ & $430(143)$ \\
Median, [QI-Q3] & $425[335-5 \mathrm{I0}]$ \\
\hline Macular volume (mm3) & \\
Mean (SD) & $10.1(2.5)$ \\
Median, [QI-Q3] & $9.8[8.9-1 \mathrm{I} .6]$ \\
\hline Qualitative SD-OCT & \\
Intraretinal cyst & $42(93.3 \%)$ \\
Sub retinal fluid & $25(55.6 \%)$ \\
Hyperreflective loci & $38(84.4 \%)$ \\
Disorganization of the retinal layer & $20(44.4 \%)$ \\
Ellipsoid zone disruption & $20(44.4 \%)$ \\
External limiting membrane disruption & $14(31.1 \%)$ \\
\hline Subfoveal choroidal thickness ( $\mu \mathrm{m})$, mean (SD) & $166(85)$ \\
\hline
\end{tabular}

Abbreviations: SD, standard deviation; SD-OCT, spectral-domain optical coherence tomography. month 6 during the loading phase: 4 cases at the second visit, 2 cases the third visit and 2 cases at the sixth visit without any further treatment. Aflibercept was switched to dexamethasone implant in 4 eyes by the investigator due to persistent of DME or because patients were reluctant to monthly injection. Aflibercept was discontinued at visit 4 in one patient and at visit 9 in another after resolution of macular edema without recurrence of DME. Finally, data was available for 34 eyes at 1 year. Among these, lapses in follow-up $\geq 2$ months due to illness or other cause, leading to unintentional treatment interruptions, occurred in $8 / 34$ eyes (24\%). The overall rate of completion of the 1 year visit was $72 \%$.

\section{Effect of Treatment on Visual Acuity}

Mean ETDRS score was $53.5 \pm 14.7$ letters (approximate Snellen equivalent 20/100) at baseline. Data was available for 36 eyes at month 6 and for 33 eyes at 1 year. Mean improvement in the visual acuity was +4.6 letters $(\mathrm{p}=$ 0.002 , CI $[+1.8 ;+7.54])$ after 5 IAI at month 6 and +6 letters $(\mathrm{p}<0.001, \mathrm{CI}[+2.7 ; 9.4])$ at 1 year. Among these, 6 eyes $(18 \%)$ gained $\geq 15$ letters, 26 eyes $(79 \%)$ remained stable (loss of $<15$ letters), and 1 eye (3\%) lost $\geq 15$ letters. Visual gain at 1 year is correlated with those at 3 months $(\mathrm{r}=0.63, \mathrm{p}=0.001, \mathrm{CI}[0.27 ; 0.87])$ and those at 6 months $(r=0.73, p<0.001)$. Gain of vision was independent of age, baseline VA, duration of DME, HbAlc and number of IAI received. We did not found difference in visual gain among treatment-naïve eyes and previously treated eyes, neither that among macula ischemia severity groups. There was a trend of worse visual gain in eyes with EZ disruption than eyes without EZ disruption (mean gain $=+3 \pm 6.7$ letters vs. $+8.9 \pm 10.8$ respectively, $\mathrm{p}=$ 0.06 ), and in eyes with external limiting membrane (ELM) disruption than eyes with preserved ELM (mean gain $=2.2$ \pm 6.9 letters vs. $7.9 \pm 10$ letters respectively, $p=0.06$ ).

\section{Effect of Treatment on Retinal Thickening} Mean central subfield thickness (CST) was $430 \pm 143 \mu \mathrm{m}$ at baseline. CST decreased by $15 \%(-65 \mu \mathrm{m}$, CI $[-107$; $-22.7], \mathrm{p}<0.001)$ at month 6 and by $25 \%(-108 \mu \mathrm{m}, \mathrm{CI}$ $[-67 ;-149], \mathrm{p}<0.001)$ at 1 year. Mean MV at baseline was $10.1 \pm 2.5 \mathrm{~mm}^{3}$. Mean reduction of MV was $-0.9 \mathrm{~mm}^{3}$ at 6 month (CI $\left.[-1.4 ; 0.4, \mathrm{p}<0.001]\right)$ and $-1 \mathrm{~mm}^{3}$ at 1 year $(\mathrm{CI}[-1.6 ;-0.4], \mathrm{p}=0.001)$. Resolution of sub retinal fluid was obtained in all eyes and persistence of intraretinal cysts was observed in 20/34 eyes (59\%). Choroidal thickness was unchanged following Aflibercept 
therapy $(166 \pm 85 \mu \mathrm{m}$ at initial examination vs. $181 \pm 91$ $\mu \mathrm{m}$ at 1 year, NS).

\section{Aflibercept Intravitreal Injections Frequency}

At month 6, 35 eyes of 46 included eyes (74\%) received as required 5 IAI every 4 weeks during the loading phase. To calculate frequency of IAI in patients who achieved the 1-year end-point, we excluded patients who abandoned treatment or who were switched to dexamethasone implant. Mean number of Aflibercept injections for 34 eyes which achieved 1 year of follow-up was $9.3 \pm 1.8$, ranging from 3 to 13 IAI for the first year. Mean interval of IAI was $5.8 \pm 3.1$ weeks.

\section{Safety}

Over the 12-month study periods, two patients (4.3\%) underwent uneventful cataract. Intraocular pressure remained stable at 1 year $(16.6 \pm 3.8 \mathrm{mmHg}$, NS). No case of endophthalmitis was reported. No serious systemic adverse event was reported during the study.

\section{Discussion}

DME treatment has evolved tremendously with the development of intravitreal anti-VEGF injection. DME surgery is considered in the presence of antero posterior traction. ${ }^{1}$ The existence of ERM in DME eyes lowered the efficacy of anti-VEGF treatment ${ }^{7}$ and PPV should be considered when the response to anti-VEGF or dexamethasone implant is incomplete. ${ }^{1}$ PPV with internal limiting membrane peeling in DME improved macular edema and visual acuity in the short term, with recurrence rate of $6 \%$ during the 6 month follow-up. ${ }^{11}$ Patients with diabetes may also undergo PPV for other reason before the occurrence of macular edema; the vitrectomy status may consequently imply change of DME management.

The pharmacokinetics of drugs injected into the vitreous in animal models ${ }^{20}$ suggest a faster clearance rate in vitrectomized eyes, particularly those with lower molecular weight. Niwa et al found that intravitreal aflibercept and ranibizumab have similar half-lives in the aqueous humor in non-vitrectomized eyes (2.3 and 2.3 days respectively) which are shorter in vitrectomized eyes (1.5 and 1.4 respectively) in macaque eyes. If the drug half-life of a biological agent, such as an anti-VEGF antibody, is shorter in a human eye after vitrectomy, there may be a shorter duration of action with less improvement and a greater need for more frequent injections over an extended time period in eyes with vitrectomy than eyes without vitrectomy.

Most of the large clinical trials of anti-VEGF drugs were performed in eyes with vitreous. Few studies have been able to compare effectiveness of anti-VEGF in vitrectomized eyes versus non-vitrectomized eyes. These studies had the retrospective nature, based on small number of patients with short duration of follow-up. Yanyali looked at 11 eyes with persistent DME despite vitrectomy and internal limiting membrane peeling treated by 3 monthly bevacizumab. The authors concluded that there was no change in VA and CST at 3 and 6 months but also wondered if continued injections for 6 or 12 months might have improved outcome. ${ }^{15}$ Koyanagi el al compared prospectively the efficacy of ranibizumab in 10 diabetic eyes with vitrectomy and 15 diabetic eyes without vitrectomy during 6 month-period. The authors found that improvement appeared to be slower, with a decrease of CST without change in VA in the vitrectomized group. ${ }^{21}$ Laugesen et al also reported reduction in CST of $14 \%$ without change in VA in a retrospective review chart of 33 vitrectomized eyes treated with ranibizumab. ${ }^{14}$ Chen et al reviewed 46 eyes previously vitrectomized at least 3 months ago, which received 3 monthly then PRN ranibizumab treatment during a follow-up at 6 months. They found no difference in effectiveness in functional and anatomical results between the vitrectomized and nonvitrectomized group, but the improvement was slower and the number of injection was higher in the vitrectomized group (5.05 vs. 4.1 injections for 6 months). ${ }^{13}$ A post hoc assessment of DME eye with previous vitrectomy in the DRCR.net trial reported the same findings than our results: these eyes had long duration of diabetes, worse visual acuity (60 letters), higher rates of previous cataract surgery (64\%), less thickened CST on OCT, worse diabetic retinopathy severity level (PDR and/or previous scatter laser in $72 \%$ ) compare to non-vitrectomized eyes. Mean improvement of VA in vitrectomized eyes was +7.7 letters and mean reduction of CST was $83 \mu \mathrm{m}$ at 1 year. Improvement appears slower and the number of ranibizumab injection was significantly higher in the vitrectomized group relative to the non-vitrectomized group (median number of 10 vs. 9 injections) during the first year, but no difference was found in anatomical response and cumulative number of injections at the 3 year endpoint. ${ }^{22}$ Overall, visual and anatomical improvement was significant in vitrectomized eyes in studies with high frequency 
ranibizumab injection: 5 injections for 6 months or 10 injections for 1 year. Compare to non-vitrectomized eyes, improvement was slower despite a higher injection frequency in vitrectomized eyes during the first year, but effectiveness was similar in both groups. ${ }^{13,22}$ In the same way, Ozsaygili et al also reported that the efficacy of Aflibercept is independent of the posterior vitreousdetachment (PVD) in DME. $^{23}$

In the APOLLON study ${ }^{24}$ including real-world patients with DME treated in France, change in VA was $+7.8 \pm 12.3$ letters in treatment-naïve patients and +5 \pm 11.3 letters in previously treated eyes with mean number of 7.6 injections. Visual gain of +4.6 letters at 6 months and of +6 letters at 1 year in our study is encouraging since these vitrectomized eyes presented multiple indicative of disease severity and worse visual prognosis of OCT biomarkers (hyperreflective foci, DRIL, EZ disruption, ELM disruption) at baseline. We did not find difference in visual gains among treatment-naïve and previously treated eyes probably because the need of vitrectomy is a marker of chronic refractory DME, and the response to aflibercept has been showed to be independent of the status of history of anti-VEGF therapy. ${ }^{2}$ These eyes also respond to Aflibercept independently to the macula ischemia severity in our study, which has been demonstrated in eyes with vitreous. ${ }^{25}$ In the present study, anti-VEGF was attempted in $76.7 \%$ among non-naïve eyes before inclusion; this suggests that, aflibercept may be used in vitrectomized eyes as first line-therapy, or second line therapy in refractory to previous antiVEGF intravitreal injection, as reported in nonvitrectomized eyes. ${ }^{6}$ Unsurprisingly, VA response to Aflibercept at 12 weeks was correlated with 1 year response in VA in our study. This individual variability in the course of vision gain as in DME treated with antiVEGF drugs and early response may predict the visual outcome. $^{26}$

The number of aflibercept injections is particularly high in this real-life study although a quarter of eyes missed a visit and treatment. Mean number of aflibercept injections was 9.3 for the 1 year period, which would be higher if there was no lapses in follow-up. Number of aflibercept injections was 8.4 to 8.7 in the VIVID and VISTA, and 10 in the DRCR.net in DME eyes with vitreous. $^{27}$ Although it is difficult to compare injection frequency among studies using different treatment protocols, the high number of Aflibercept injections and shorter interval injection found in this study may be explained by the increase clearance of the drug post vitrectomy, as consequent, the need to adopt the injection frequency to achieve improvement. This high frequency in the human model of our study is consistent with findings of Niwa and al who found that Aflibercept suppressed VEGF levels during only 4 weeks in vitrectomized macaque eyes. ${ }^{16}$ Based on results of pharmacokinetics of intravitreally administrated Aflibercept in animal models and results of our study in human models, we would recommend a fixed monthly injection regimen, closed monitoring until improvement of visual acuity and resolution of macula edema.

So far, this is the first study of Aflibercept therapy in vitrectomized eyes with DME in human. There are several limitations that need to be addressed. The conversion of Snellen chart converted to ETDRS letter scores may be a potential bias. Observational real-world open studies encompassed bias of dropped out patients and break-up visit and treatment due to comorbidities. We did not plan a control group because of imbalances between the vitrectomy and non-vitrectomy group at baseline with potential cofounders that could affect the outcome. The study has the strength of a prospective design with homogenous treatment regimen and follow-up. Further study is needed to evaluate long term results of aflibercept in these particular eyes.

\section{Conclusion}

In conclusion, in this prospective study, that aflibercept intravitreal injection is effective in previously vitrectomized eyes with DME, leading to visual and anatomical gain, using higher frequency than $2 \mathrm{q} 8$. These eyes may require more consistent monthly Aflibercept treatment during the first year. Our results will be a useful addition to armamentarium in the treatment of DME in this particular group.

Institutional Review Board approval: The study was approved by the French National Medical Council.

Meeting: The abstract of this study was presented in ARVO 2020, Baltimore, May $3-7^{\text {th }}$. The poster's abstract was published in Investigative Ophthalmology \& Visual Science June 2020, Vol.61, 3307.

\section{Precis}

Aflibercept intravitreal injections may be beneficial in vitrectomized eyes with refractory persistent DME which require frequent injection to obtain visual gain and reduction of CST. 


\section{Key Messages}

What is already known: There is little information with inconsistent results in vitrectomized eyes with DME treated with ranibizumab and bevacizumab. No information is available on efficacy of Aflibercept in this particular group.

What are the new findings: Aflibercept is effective in previously vitrectomized eyes with DME using higher frequency than $2 \mathrm{q} 8$ at one year.

How might these results change the focus of research or clinical practice: The results suggest the need of fixed monthly Aflibercept injection regimen in vitrectomized eyes with DME.

\section{Author Contributions}

All authors made substantial contributions to conception and design, acquisition of data, or analysis and interpretation of data; took part in drafting the article or revising it critically for important intellectual content; agreed to submit to the current journal; gave final approval of the version to be published; and agree to be accountable for all aspects of the work.

\section{Funding}

The first author got the funding from Eurasante call for projects of the North of France Region. The Medical Research Department of Lille Catholic Hospitals received funding from Bayer Healthcare SAS to run the study. The sponsor had no role in the design or conduct of this research.

\section{Disclosure}

Prof. Dr. Thi Ha Chau Tran reports consultancy for Bayer Healthcare, and personal fees from Novartis, Allergan during the conduct of the study. Dr Ali Erginay reports non-financial support from Bayer, Novartis, outside the submitted work. Dr Joel Uzzan reports personal fees from Allergan and being a consultant and investigator for Bayer and Novartis, outside the submitted work. Prof. Stephanie Baillif reports consultancy for Bayer, Novartis, Allergan, and Horus Pharma, during the conduct of the study. Prof. Dr. Laurent Kodjikian reports personal fees from Bayer, Allergan, Novartis, and Roche, during the conduct of the study. The authors report no other potential conflicts of interest for this work and report no proprietary or commercial interest in any materials discussed in this article.

\section{References}

1. Schmidt-Erfurth U, Garcia-Arumi J, Bandello F, et al. Guidelines for the Management of Diabetic Macular Edema by the European Society of Retina Specialists (EURETINA). Ophthalmologica. 2017;237(4):185-222. doi:10.1159/000458539

2. Do DV, Nguyen QD, Vitti R, et al. Intravitreal aflibercept injection in diabetic macular edema patients with and without prior anti-vascular endothelial growth factor treatment: outcomes from the phase 3 Program. Ophthalmology. 2016;123(4):850-857. doi:10.1016/j. ophtha.2015.11.008

3. Korobelnik JF, Do DV, Schmidt-Erfurth U, et al. Intravitreal aflibercept for diabetic macular edema. Ophthalmology. 2014;121 (11):2247-2254. doi:10.1016/j.ophtha.2014.05.006

4. Heier JS, Bressler NM, Avery RL, et al. Comparison of aflibercept, bevacizumab, and ranibizumab for treatment of diabetic macular edema: extrapolation of data to clinical practice. JAMA Ophthalmol. 2016;134(1):95-99. doi:10.1001/jamaophthalmol.2015.4110

5. Wells JA, Glassman AR, Ayala AR, et al. Aflibercept, Bevacizumab, or Ranibizumab for Diabetic Macular Edema: two-Year Results from a Comparative Effectiveness Randomized Clinical Trial. Ophthalmology. 2016;123(6):1351-1359. doi:10.1016/j.ophtha.2 016.02.022

6. Bahrami B, Hong T, Schlub TE, Chang AA. AFLIBERCEPT FOR PERSISTENT DIABETIC MACULAR EDEMA: forty-Eight-Week Outcomes. Retina. 2019;39(1):61-68. doi:10.1097/IAE.00000000 00002253

7. Hagenau F, Vogt D, Ziada J, et al. Vitrectomy for diabetic macular edema: optical coherence tomography criteria and pathology of the vitreomacular interface. Am $J$ Ophthalmol. 2019;200:34-46. doi:10.1016/j.ajo.2018.12.004

8. Kulikov AN, Sosnovskii SV, Berezin RD, Maltsev DS, Oskanov DH, Gribanov NA. Vitreoretinal interface abnormalities in diabetic macular edema and effectiveness of anti-VEGF therapy: an optical coherence tomography study. Clin Ophthalmol. 2017;11:1995-2002. doi:10.2147/OPTH.S146019

9. Ghassemi F, Bazvand F, Roohipoor R, Yaseri M, Hassanpoor N, Zarei M. Outcomes of vitrectomy, membranectomy and internal limiting membrane peeling in patients with refractory diabetic macular edema and non-tractional epiretinal membrane. J Curr Ophthalmol. 2016;28 (4):199-205. doi:10.1016/j.joco.2016.08.006

10. Haller JA, Qin H, Apte RS, et al. Vitrectomy outcomes in eyes with diabetic macular edema and vitreomacular traction. Ophthalmology. 2010;117(6):1087-1093 e1083.

11. Michalewska Z, Stewart MW, Landers MB 3rd, Bednarski M, Adelman RA, Nawrocki J. Vitrectomy in the management of diabetic macular edema in treatment-naive patients. Can $j$ Ophthalmol. 2018;53(4):402-407. doi:10.1016/j.jcjo.2017.10.011

12. Chen YY, Chang PY, Wang JK. Intravitreal aflibercept for patients with diabetic macular edema refractory to bevacizumab or ranibizumab: analysis of response to aflibercept. Asia Pac J Ophthalmol. 2017;6(3):250-255. doi:10.22608/APO.2016186

13. Chen YY, Chen PY, Chen FT, Chen YJ, Wang JK. Comparison of efficacy of intravitreal ranibizumab between non-vitrectomized and vitrectomized eyes with diabetic macular edema. Int Ophthalmol. 2018;38(1):293-299. doi:10.1007/s10792-017-0462-1

14. Laugesen CS, Ostri C, Brynskov T, et al. Intravitreal ranibizumab for diabetic macular oedema in previously vitrectomized eyes. Acta Ophthalmol. 2017;95(1):28-32. doi:10.1111/aos.13160

15. Yanyali A, Aytug B, Horozoglu F, Nohutcu AF. Bevacizumab (Avastin) for diabetic macular edema in previously vitrectomized eyes. Am J Ophthalmol. 2007;144(1):124-126. doi:10.1016/j. ajo.2007.02.048 
16. Niwa Y, Kakinoki M, Sawada T, Wang X, Ohji M. Ranibizumab and aflibercept: intraocular pharmacokinetics and their effects on aqueous VEGF level in vitrectomized and nonvitrectomized macaque eyes. Invest Ophthalmol Vis Sci. 2015;56(11):6501-6505. doi:10.1167/ iovs.15-17279

17. Avitabile T, Azzolini C, Bandello F, et al. Aflibercept in the treatment of diabetic macular edema: a review and consensus paper. Eur J Ophthalmol. 2017;27(6):627-639. doi:10.5301/ejo.5001053

18. Liu S, Wang D, Chen F, Zhang X. Hyperreflective foci in OCT image as a biomarker of poor prognosis in diabetic macular edema patients treating with Conbercept in China. BMC Ophthalmol. 2019;19 (1):157. doi:10.1186/s12886-019-1168-0

19. Choi MY, Jee D, Kwon JW. Characteristics of diabetic macular edema patients refractory to anti-VEGF treatments and a dexamethasone implant. PLoS One. 2019;14(9):e0222364. doi:10.1371/journal.pone.0222364

20. Kakinoki M, Sawada O, Sawada T, Saishin Y, Kawamura H, Ohji M. Effect of vitrectomy on aqueous VEGF concentration and pharmacokinetics of bevacizumab in macaque monkeys. Invest Ophthalmol Vis Sci. 2012;53(9):5877-5880. doi:10.1167/iovs.12-10164

21. Koyanagi Y, Yoshida S, Kobayashi Y, et al. Comparison of the Effectiveness of Intravitreal Ranibizumab for Diabetic Macular Edema in Vitrectomized and Nonvitrectomized Eyes. Ophthalmologica. 2016;236(2):67-73. doi:10.1159/000446992
22. Bressler SB, Melia M, Glassman AR, et al. Ranibizumab plus prompt or deferred laser for diabetic macular edema in eyes with vitrectomy before anti-vascular endothelial growth factor therapy. Retina. 2015;35(12):2516-2528. doi:10.1097/IAE.0000000000000617

23. Ozsaygili C, Kucuk B, Yildirim Y. The effect of posterior vitreous detachment on aflibercept response in diabetic macular oedema. $\mathrm{Br}$ J Ophthalmol. 2020. doi:10.1136/bjophthalmol-2020-316155

24. Korobelnik JF, Daien V, Faure C, et al. Real-world outcomes following 12 months of intravitreal aflibercept monotherapy in patients with diabetic macular edema in France: results from the APOLLON study. Graefe Arch Clin Exp Ophthalmol. 2020;258(3):521-528. doi:10.1007/s00417-019-04592-9

25. Wykoff CC, Shah C, Dhoot D, et al. Longitudinal retinal perfusion status in eyes with diabetic macular edema receiving intravitreal aflibercept or laser in VISTA Study. Ophthalmology. 2019;126 (8):1171-1180. doi:10.1016/j.ophtha.2019.03.040

26. Koyanagi Y, Yoshida S, Kobayashi Y, et al. Visual outcomes based on early response to anti-vascular endothelial growth factor treatment for diabetic macular edema. Ophthalmologica. 2018;239(2-3):94-102. doi:10.1159/000481711

27. Wells JA, Glassman AR, Ayala AR, et al. Aflibercept, bevacizumab, or ranibizumab for diabetic macular edema. $N$ Engl J Med. 2015;372 (13):1193-1203.
Clinical Ophthalmology

\section{Publish your work in this journal}

Clinical Ophthalmology is an international, peer-reviewed journal covering all subspecialties within ophthalmology. Key topics include: Optometry; Visual science; Pharmacology and drug therapy in eye diseases; Basic Sciences; Primary and Secondary eye care; Patient Safety and Quality of Care Improvements. This journal is indexed on PubMed

\section{Dovepress}

Central and CAS, and is the official journal of The Society of Clinical Ophthalmology (SCO). The manuscript management system is completely online and includes a very quick and fair peer-review system, which is all easy to use. Visit http://www.dovepress.com/ testimonials.php to read real quotes from published authors. 